\title{
The Research of Human-computer Interactive Terminal Design for the
}

\section{Nurse Information System}

\author{
Yihong Wang ${ }^{1,}$, , Lili Liu ${ }^{2, b^{*}}$ \\ 1 Jiangsu Key Laboratory of Large Engineering Equipment Detection and Control, Xuzhou Institute \\ of Technology, Xuzhou, Jiangsu Province, 221000,China \\ 2 Jiangsu Key Laboratory of Large Engineering Equipment Detection and Control, Xuzhou Institute \\ of Technology, Xuzhou, Jiangsu Province, 221000,China \\ a18052120752@sina.com, bdxwll@@sina.com
}

Keyword: Human-computer Interaction. Nurses' Information. Terminal Design. Form Design

Abstract. This paper mainly analyzes the nurse information system stream and hardware configuration accordingly. In the light of the behavior and need of nurses and patients, this design sets human-computer interaction for the nurses' information, proposes the concept of its information terminal, and does some user-friendly study. And eventually, market surveys and semantic differential working out the product conformation intent, the primary product pattern is designed.

The nurse information terminal is the nurse terminal of clinical information system and meanwhile, the end-execution system convenient to carry on the wards, whose primary users are nurses working on inpatient wards, and operating environment is the wards inside, with the effect of optimizing and simplifying nurses' working process, collecting and holding patients' information, facilitating a timely and effective communication between doctors and patients.

\section{The Function Setting of Nurse-information Human-machine Interface}

\section{Information Display(\&Query) Function}

a. Patient's basic information display(\&query).Including patient's hospital number, bed number, name, sex, age, time of being admitted into the Ward, clinical department, diagnosis, attending doctor, state of disease, dieting, nursing level, weight, height, time of operation, allergies, expense and so forth.

b. Patient's vital signs display(\&query).Including collected information like body temperature, blood pressure, heart rate, breathing, pulse and so forth , and generating temperature chart, patient's vital signs observation sheet, nursing records and so forth. Patient's biochemical data recorded one more time, the system would show a curve of historical data, which directly reflecting the trend of the change state of illness.

c. Doctor's advice displays(\&query).Doctor's advice is split into every patient's information data series by clinical pathway. Hardly when receiving a new doctor's advice, the system would auto-suggest, and execute the doctor's advice needed only the very day, so as to improve the efficiency

d. Nursing working plan display. Work needed finishing the very day, how the care a patient need, there would be a detailed arrangement and a clear remind.

e. Prescription displays. 
f. Caller ID displays. When patient or nurse accepts a call, the system shows where the patient's hospital room and bed number are, in order to identify the source of the information quickly.

\section{Information recording function}

Mainly for many kinds of text information recording, as a port for information gathering.

Patient's vital signs information recorded. The system would record the result of test and measurement promptly and accurately[1]. Typing in the content in standard forms, including body temperature, blood pressure, heart rate, breathing, pulse and something more about the patient's basic information, and the record of nursing service, filling in nursing sheets, filling in prescriptions, patient's observations, and many kinds of inspection records and so on.

\section{Record timers function}

Record timers function includes timing function, memory function, voice informer function and visual reminder function.

Where to use the record timer function:

Arrange the nursing project to every patient reasonably, and regularly remind a nurse of finding which patient to do what job. As for the inspection project in stipulated time, the nurse can preset a time and make time for other jobs [2].

The Function of Nurse information Terminator and its Construction Drawing

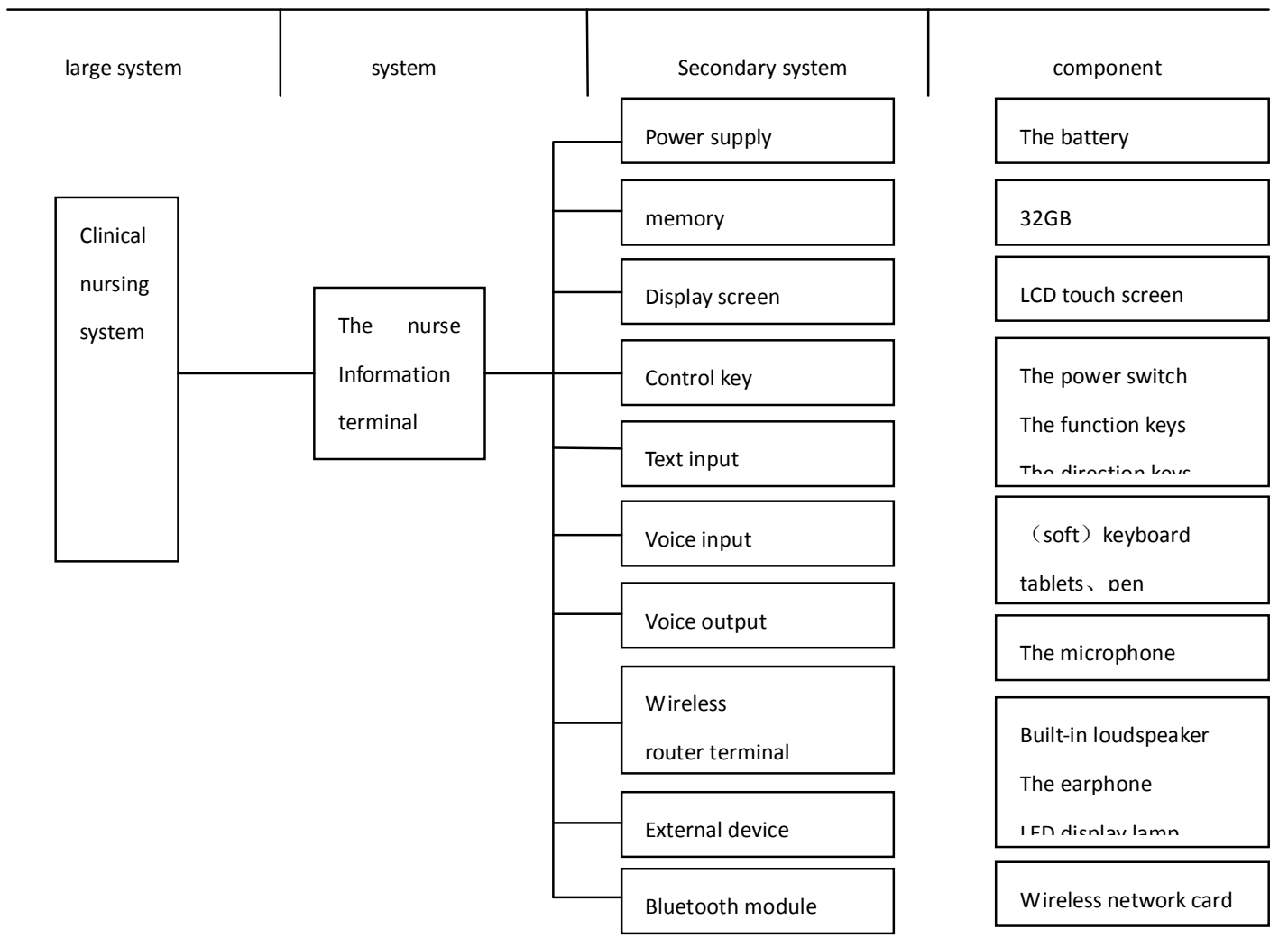

Figure 1 the Function of Nurse information Terminator and its Construction Drawing

\section{.Nurse information terminal modeling intention analysis}

\section{Research method selection}

First of all,in order to know exactly the variable of the nurse-to-information-terminal-product sensibility, a system should design the terminal product meeting the nurse's requirements much more[3]. It'd better to choose SD to investigate and analyze the variable of the nurse-to-product sensibility. Semantic differential method's application is used to realize the trend of product concept and modeling idea, usually seen as a important method to evaluate the immeasurable data. This 
means could help to comprehend the nurse-to-information-terminal sensible acknowledge and styles and preference, in which way the product meet the nurses' usage habits.

Secondly, data an alysis: analyze the results after finishing the questionnaire. Mathematical statistical methods mainly uses the cluster analysis, questionnaires and some other methods to confirm the level of similarity between perceptual adjectives, based on which grouping the perceptual adjectives and selecting representative perceptual adjectives. By analyzing the results, the trend of product image that the nurses' adjective corresponding to is concluded. Eventually the perceptual concept of product patterns is formed[4].

\section{Research starting}

1). Questionnaire's setting

a. Gather and select perceptual adjectives for demonstrating the information product

b. Select three information terminal products with great differences(represented by mobile phones), as the picture showed

c. Establish semantic differential variable table: establish the 7th differential variable table composed by the final selected perceptual adjectives and 3 different kinds of mobile phone products
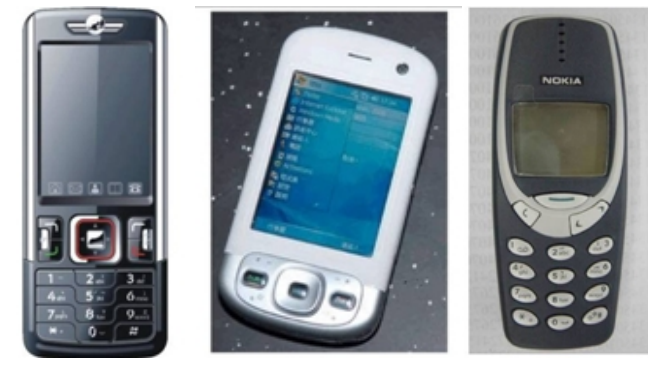

Figure 2 product1,2,3

\section{The results of the survey analysis}

Analysis the date of the above SD form qualitatively: first, calculated for the average that each sample corresponds to the emotional vocabulary and generate the chart shown in figure 3 through the EXCEL software. Then, analysis the date according to the chart quantitatively.

\begin{tabular}{|c|c|c|c|c|c|c|c|c|c|}
\hline & & har & outmo & $\mathrm{w}$ & com & masc & oldn & Low & sl \\
\hline & $\mathrm{d}$ & & ded & $\mathrm{rm}$ & plex & uline & ess & profile & ick \\
\hline prod & & 2.27 & -2.16 & -1 & 0.35 & 2.16 & -2.0 & 0.16 & - \\
\hline uct1 & & & & 98 & & & 2 & & 1.78 \\
\hline prod & & -1 & -2.23 & -0 & -1.2 & -1.58 & -2.1 & 0.53 & - \\
\hline uct2 & 23 & & & 34 & 7 & & 8 & & 2.03 \\
\hline prod & & -0 & 2.03 & -1 & 0.93 & 1.99 & 2.01 & 0.12 & 1 \\
\hline \multirow[t]{3}{*}{ uct3 } & 21 & & & 78 & & & & & .98 \\
\hline & & har & outmo & w & com & masc & oldn & Low & sl \\
\hline & d & & ded & $\mathrm{rm}$ & plex & uline & ess & profile & ick \\
\hline prod & & 2.27 & -2.16 & -1 & 0.35 & 2.16 & -2.0 & 0.16 & - \\
\hline uct1 & & & & 98 & & & 2 & & 1.78 \\
\hline prod & & -1 & -2.23 & -0 & -1.2 & -1.58 & -2.1 & 0.53 & - \\
\hline uct2 & 23 & & & 34 & 7 & & 8 & & 2.03 \\
\hline prod & & -0 & 2.03 & -1 & 0.93 & 1.99 & 2.01 & 0.12 & 1 \\
\hline uct3 & 21 & & & 78 & & & & & .98 \\
\hline
\end{tabular}

Figure 3 SD Data 
We can find it's best interpretation of the element design through the analysis which counting the Characteristics of the product samples and corresponding emotional vocabulary scores .Such as ,the emotional vocabulary "modern", the highest score is the product sample 1 and 2 , on the contrary, sample 3 gets low scores. We can find some corresponding rules between the two through the characteristics of the product samples and emotional vocabulary. Every emotional appeals reaction on three aspects :the overall product, interface, the button .Further said that product overall, including form, color, material; the color, typography, software system in the interface; the form, color, material in the button and the use of products. For "modern information terminal" design elements combination can be summarized roughly the hale appearance, simple key combination, clear interface combination, input mode of humanity etc.

\section{Form Design of Nurse Information Terminator}

According to the characteristics of the research and nurses, form design as shown in figure 4.

Form design of nurse Information terminator using soft and thin body shape, choose four inch touch screen, the whole reference size is $90 * 50 * 12 \mathrm{~mm}$. Function buttons only include guide buttons and answer hangs up keys in order to reducing operation. Meanwhile, main information input and query work are conducted on a touch screen. The edge of product using the non-slip drop rubber material. Larger screen and buttons are used for nurses[5].

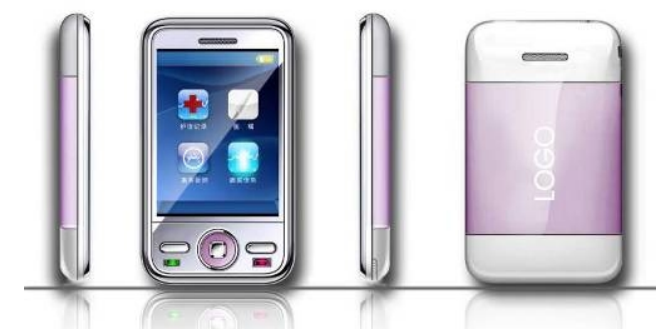

Figure 4 Nurse Information Terminator Sketch

Also comes with a stylus and rope buckle for writing and carry. Color with light tone of pink in the white basic conform to the aesthetic intention female nurses[6].

\section{Conclusion}

The major function of nurse Information terminator is to collect and store patient information, accept and execute orders, do nursing records, communicate with patients etc. Mainly used for record and query the patient information, real time observation and record the patient vital signs, generate related record form automatically, proofread and executed the doctor's advice and care work arrangements, improve ward exchanges of communication for not finishing work.

In a word, clinical nurse Information terminator is a kind of intelligent medical care system which based on people-centered concept. It will change the way of medical staff work, making it more convenient and more human.

\section{Reference:}

[1] Huaping Liu. The nurse human resources present status analysis and standard research[J].China's nursing management,2005,5(4):25.

[2] Chenghua Huang et al."Taking patient as the center": the core concept of the harmonious doctor-patient relation[J].Chinese medical ethics,2008,21 (6): 52-53 
[3] Yulin Chai. Build a patient-centered hospital information management system[J].Medical information, 2008,21(6):789.

[4] Xinghua Zhang et al. Talk about digital medical[J]. Medical equipment information,2002.6:35.

[5]. Haiyan Qi, Hong Wu, Fengxian Yang. The disadvantages and countermeasures of entering the doctor's advice by nurse computer[J]. Chinese journal of practical nursing,2005,21(6):66.

[6] Ying Wang,Guoben Zhao. Overview of qualitative research method[J]. Foreign medical·Social medical layered,2004,21(4):178. 